Revista

Multi-Ensayos

Vol. 4, $\mathrm{N}^{\circ} 8$

ISSN: 2412-3285

https://multiensayos.unan.edu.ni

DOI: https://doi.org/10.5377/multiensayos.v4i8.9447

\title{
Aprovechamiento del bono demográfico para emprender en unidad el desarrollo próspero de Nicaragua
}

\section{Use of the demographic bonus in order to undertake in unity the development of Nicaragua}

\author{
Bladimir Caldéron Aguilera ${ }^{1}$
}

Recibido: 6 de noviembre de 2018, Aceptado: 12 de febrero de 2019

\section{RESUMEN}

El presente escrito surge a partir de la decisión del autor de participar en el concurso nacional de ensayos: Ideas juveniles para el desarrollo nacional, convocado por el Ministerio de la Juventud (MINJUVE), durante el mes de octubre, en el cual una de las temáticas propuestas para la elaboración del ensayo era la dinámica demográfica, en este caso particular profundizando en el tema del bono demográfico en Nicaragua. Es por ello que a medida que se avance en la lectura del documento se encontrara una breve explicación de la importancia de la temática abordada, así mismo se brindara la definición de bono demográfico, datos del bono demográfico en Nicaragua, incorporación del tema en aspectos gubernamentales y algunas ideas para el aprovechamiento del bono demográfico en el desarrollo nacional, finalizando con las conclusiones del escrito, y la bibliografía consultada.

Palabras claves: desarrollo; juventud; bono demográfico; gobierno de Nicaragua.

\section{ABSTRACT}

This paper merged from the writer's decision of participating in the National Contest of Essays: "Youth ideas for the national development", guided by the Youth Minister (MINJUVE), in October 2018. One of the topics was the demography, specifically the demographic bonus in Nicaragua. This paper gathers a brief explanation of the importance of the topic, the definition and data about the demographic bonus in the country, also its incorporation in the governmental aspects and some ideas to use in the national development.

Keywords: development; yuth; demographic bonus; goverment of Nicaragua.

1 Profesor de Educación Media en el Instituto Nacional Francisco Luis Espinoza (INFLE), Correo electrónico: bladicalde1993@ gmail.com

Copyright (c) 2018 Revista Multi-Ensayos.

(c) (i) (2) Este trabajo está licenciado bajo una Licencia Internacional Creative Commons 4.0 Atribución-NoComercial-Compartirlgual. 


\section{INTRODUCCIÓN}

Un tema relevante de la dinámica demografía, es el bono demográfico, y Nicaragua en este contexto presenta un momento único para su aprovechamiento, para ello este ensayo pretende:

- Describir aspectos generales del bono demográfico

- Presentar algunas formas de inclusión del tema bono demográfico en la agenda del Gobierno de Reconciliación y Unidad Nacional.

- Brindar ideas para su aprovechamiento en ámbitos socioeconómicos en Nicaragua.

Para efecto del cumplimiento de los objetivos propuestos en el desarrollo del ensayo se abordará, la definición de bono demográfico, población total según grandes grupos etareos y sexo, inclusión del tema de juventud en el Plan Nacional de Desarrollo Humano (PNDH) e ideas para aprovechar el bono demográfico en los aspectos socioeconómicos del país.

\section{DESARROLLO}

Para iniciar es necesario conocer ¿Qué es bono demográfico? Al respecto se dice que es "una fase en que el equilibrio entre edades resulta una oportunidad para el desarrollo" (Centro Latinoamericano y Caribeño de Demografía (CELADE), División de población, Comisión Económica Para América Latina (CEPAL), 2008, p. 17).

En el caso de Nicaragua según la gráfica denominada población total según grandes grupos etareos y sexo (abajo presentada) el 63\% de la población nacional está entre los rangos de edades de 15 a 64 años, y un $37 \%$ de la población se encuentra en rangos menores de 14 años y mayores de 65, lo que implica que la mayor parte de la Población es parte de la Población Económicamente Activa, y el mayor porcentaje lo representan las mujeres.

\section{POBLACIÓN TOTAL SEGÚN GRANDES GRUPOS ETAREOS Y SEXO}

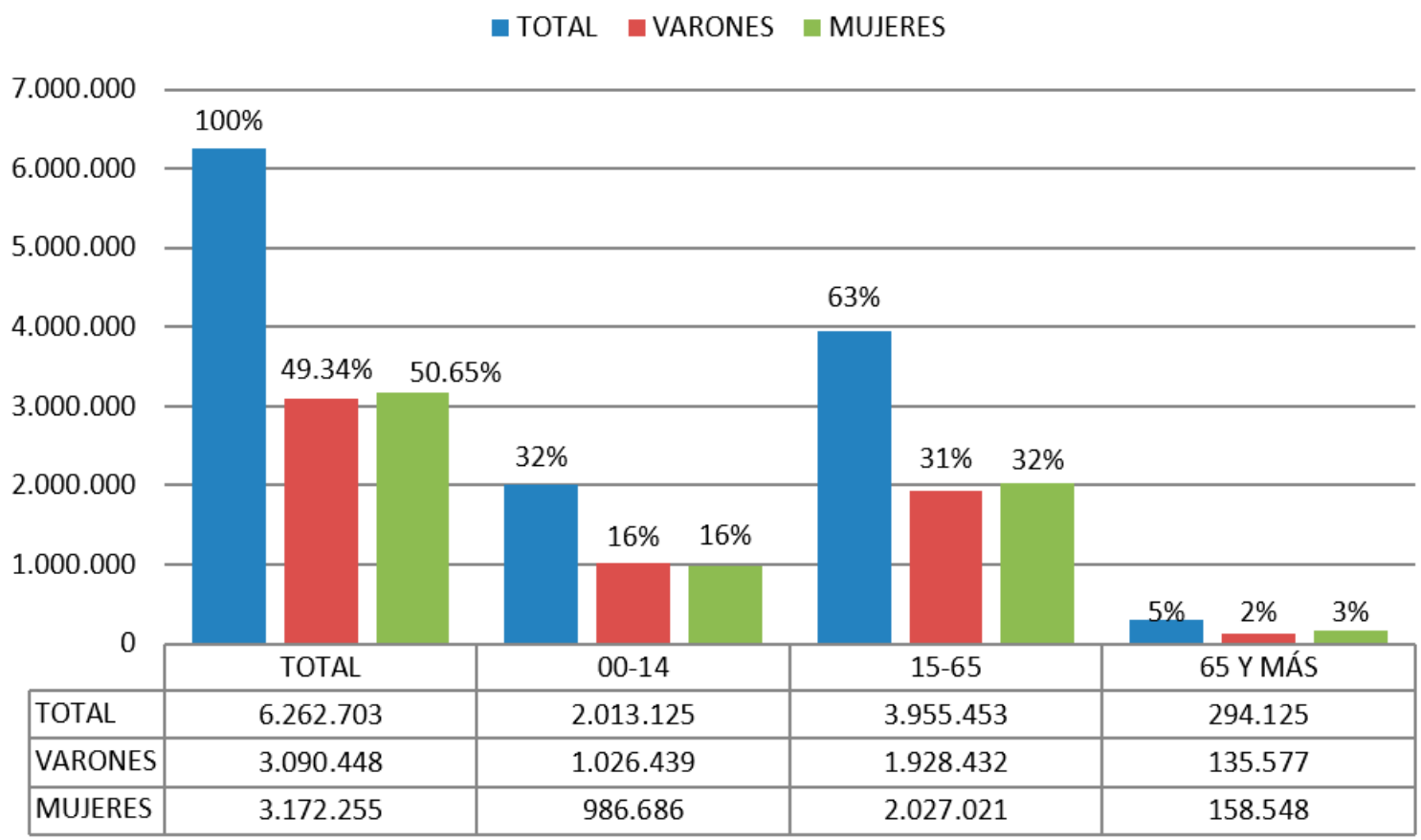

Fuente: Elaboración propia a partir de datos del Instituto Nacional de Información de Desarrollo- INIDE (2016, pág. 38). 
Y precisamente el Gobierno de Reconciliación y Unidad Nacional de la República de Nicaragua (GRUN), consciente desde siempre del rol protagónico de la JUVENTUD en diferentes ámbitos, incluye en los ejes del Plan Nacional Desarrollo Humano (PNDH) en el acápite denominado juventud y adolescencia lo siguiente: se debe "Consolidar la participación y protagonismo de la juventud para el desarrollo económico, político y social de nuestro país" (Consejo de Comunicación y Ciudadanía, 2017, p. 23), rol de importancia en este contexto social y demográfico.

Algunas ideas para aprovechar el bono demográfico en los aspectos Socioeconómicos de Nicaragua son:

1. En primera instancia se debe mencionar que, "El conocimiento tradicional entendido como datos e información para ser almacenado, está cediendo terreno al nuevo concepto del conocimiento que es acción e innovación." (Gobierno de Nicaragua, Ministerio de Educación (MINED), Instituto Tecnológico Nacional (INATEC), Consejo Nacional de Universidades (CNU), 2017, p. 16), por lo cual considero importante el levantamiento de información de jóvenes productores, emprendedores, técnicos y profesionales para que sean atendidos a través de las instituciones públicas u otras entidades, respondiendo a las necesidades de acuerdo a la información obtenida.

2. Otro elemento importante es continuar "fortaleciendo y desarrollando nuestro Modelo Constitucional de Alianzas, Diálogo y Consensos, a Nivel Nacional y Local" (Murillo Zambrana, 2017), mediante la promoción y conformación de asociaciones juveniles de egresados técnicos y/o profesionales para creación de micro/pequeñas empresas que puedan acceder a proyectos de financiamiento reembolsable a mediano o largo plazo. dos ejemplos, prácticos de asociación de estudiantes técnicos próximos a egresar serian:

a. un/a técnico/a en contabilidad, un/a soldador/a, un/a electricista

b. un/a técnico/a en repostería, comidas nicaragüenses, y comidas y gastronomía.

5. También para fortalecer el modelo de Alianza, diálogo y consensos, y el emprendedurismo, se podrían organizar ferias juveniles presentando proyectos socioeconómicos innovadores, para que instituciones gubernamentales, otras entidades y dueños de emprendimientos puedan interesarse en el desarrollo de su negocio (comprando la idea desarrollada) y a la vez facilitar empleos (temporales o permanentes) a los jóvenes que proponen las ideas.

6. Así mismo, es meritorio destacar que "la educación es considerada una prioridad para la transformación económica, social, ambiental y humana de Nicaragua" (Talavera, 2017). Al respecto, la siguiente tabla denominada cantidad de personas matriculadas en las diferentes instituciones educativas de Nicaragua durante el año 2015 muestra que la tasa neta de escolarización disminuye a medida que las personas avanzan en sus grados académicos, de primaria a secundaria se observa una disminución de 12 puntos porcentuales, y el acceso la cobertura y acceso a la Educación técnica se hace aun más baja, de igual forma sucede con la Educación universitaria.

Para afrontar esa realidad educativa nacional se propone realizar capacitaciones, talleres, encuentros y conferencias para abordar con jóvenes de diversos sectores (sobre todo con jóvenes de secundaria y jóvenes bachilleres que han descontinuado sus estudios) abordando la importancia de la permanencia escolar y la continuidad Educativa (técnica y profesional), independientemente si la labor a la que desean acceder es trabajo por cuenta propia, a nivel familiar, asalariado, cooperativismo. 


\begin{tabular}{|c|c|c|}
\hline \multicolumn{3}{|c|}{$\begin{array}{r}\text { Cantidad de personas matriculadas en } \\
\text { diferentes instituciones educativas } \\
\text { Nicaragua durante el año } 2015\end{array}$} \\
\hline $\begin{array}{l}\text { Ministerio de } \\
\text { Educación } \\
\text { (MINED) }\end{array}$ & $\begin{array}{c}\text { Instituto } \\
\text { Tecnológico } \\
\text { Nacional } \\
\text { (INATEC) }\end{array}$ & $\begin{array}{c}\text { Consejo } \\
\text { Nacional de } \\
\text { Universidades } \\
\text { (CNU) }\end{array}$ \\
\hline $1,824,300$ & 393,514 & 166,512 \\
\hline $\begin{array}{l}\text { Total de } \\
\text { matricula }\end{array}$ & \multicolumn{2}{|c|}{$2,384,326$} \\
\hline $\begin{array}{l}\text { Porcentaje de } \\
\text { la tasa neta }\end{array}$ & $\begin{array}{l}\text { Primaria } \\
\text { Regular }\end{array}$ & $\begin{array}{c}\text { Secundaria } \\
\text { Regular }\end{array}$ \\
\hline $\begin{array}{c}\text { de } \\
\text { escolarizacion }\end{array}$ & $93 \%$ & $81 \div$ \\
\hline
\end{tabular}

Fuente: (Informe Anual del Presidente de la República al Pueblo Nicaragüeense y a la Asamblea Nacional, 2015, pp. 40-53)

\section{CONCLUSIONES}

En síntesis, Nicaragua está en su mejor momento para que la juventud asuma un rol, aún más protagónico en el desarrollo del país y desde diversos ámbitos, por lo tanto, la inversión en educación y en el ámbito laboral debe seguirse trabajando con más ahínco y de manera permanente, para brindar mayores posibilidades de acceso a la población joven nicaragüense.

De igual forma, es necesario mencionar que las ideas propuestas pueden ser utilizadas como base para desarrollar proyectos y/o programas a nivel gubernamental, Organizaciones No Gubernamentales (ONG's) y otras entidades.

\section{BIBLIOGRAFÍA}

Centro Latinomericano y Caribeño de Demografía (CELADE), División de población, Comisión Económica Para América Latina (CEPAL). (2008, Noviembre). Comisión Ecnomómica Para América Latina. (P. Saad, T. Miller, C. Martinez, \& M. Holz, Eds.) Retrieved Septiembre 30, 2018 from https://www. cepal.org/celade/noticias/documentosdetrabajo/2/37142/OIJ-CELADEBono_dem.pdf

Consejo de Comunicación y Ciudadanía. (2017, Diciembre). El 19 digital. Retrieved Septiembre 30, 2018 from https://www.el19digital.com/app/webroot/tinymce/source/2018/00-Enero/ Del22al28Enero/Viernes26Enero/EJES\%20DEL\%20PROGRAMA\%20NACIONAL\%20DE\%20 DESARROLLO\%20HUMANO.pdf

Gobierno de Nicaragua, Ministerio de Educación (MINED), Instituto Tecnológico Nacional (INATEC), Consejo Nacional de Universidades (CNU). (2017, Septiembre). El Paradigma Educativo, Congreso UALN. Retrieved Octubre 10, 2018 from http://www.congresoualn.cnu.edu.ni/documentos/ resumen/lib_resumen_vf.pdf

Informe Anual del Presidente de la República al Pueblo Nicaraguense y a la Asamblea Nacional. (2015). (E. 1. digital, Ed.) Retrieved Octubre 10, 2018 from https://www.el19digital.com/articulos/ver/ titulo:38897-gobierno-sandinista-presenta-informe-anual-2015-a-la-asamblea-nacional

Instituto Nacional de Información de Desarrollo (INIDE). (2016). Retrieved septiembre 30, 2018 from http://www.inide.gob.ni/Anuarios/Anuario\%20Estadistico\%202016.pdf 
Murillo Zambrana, R. (2017, Diciembre 30). Caminante no hay camino camino, se hace camino al andar! Managua.

Talavera, T. (2017, Febrero 06). El 19 digital. Retrieved Octubre 10, 2018 from La Revolución Educativa en Nicaragua: https://www.el19digital.com/articulos/ver/titulo:51938-la-revolucion-educativaen-nicaragua 